\title{
Combining ant colony optimization with 1-opt local search method for solving constrained forest transportation planning problems
}

\author{
Pengpeng Lin*1, Ruxin Dai ${ }^{2}$, Marco A. Contreras ${ }^{3}$, Jun Zhang ${ }^{4}$ \\ ${ }^{1}$ Department of Mathematics, Statistics and Computer Science, University of Wisconsin Stout, Menomonie WI, United States \\ ${ }^{2}$ Department of Computer Science and Information System, University of Wisconsin River Falls, River Falls WI, United States \\ ${ }^{3}$ Department of Forestry, University of Kentucky, Lexington $K Y$, United States \\ ${ }^{4}$ Department of Computer Science, University of Kentucky, Lexington KY, United States
}

Received: February 7, 2017

DOI: $10.5430 / a i r . v 6 n 2 p 27$
Accepted: March 29, $2017 \quad$ Online Published: April 4, 2017

URL: https://doi.org/10.5430/air.v6n2p27

\begin{abstract}
We developed a two-stage approach (ACOLS) combining the ant colony optimization (ACO) algorithm and a 1-opt local search to solve forest transportation planning problems (FTPPs) considering fixed and variables costs and sediment yields expected to erode from road surfaces as side constraints. The ACOLS was designed for improving ACO performance and ensure the applicability to real-world, large-scale FTPPs with multiple time periods. It consists of three major routines: i) least-cost route finding process from all timber sales simultaneously, ii) two stage search process developed to quickly find feasible (stage I) and high-quality (stage II) solutions and, iii) 1-opt local search solution refinement to further improve solution quality. The ACOLS was first applied to a medium-scale hypothetical FTPP on which four cases with increasing level of sediment constraint were considered. To test for robustness, the ACOLS was then applied to ten different problems instances created basing on the same topology of the hypothetical FTPP. Lastly, the ACOLS was applied to a real-world, large-scale FTPP considering thousands of roads segments, hundreds of timber sales, and multiple products and planning periods. Feasible solutions were found for all cases indicating the usefulness of our approach to provide managers with an efficient tool to address large-scale transportation problems.
\end{abstract}

Key Words: Transportation, Ant colony optimization, Forest operations, Mixed integer programming

\section{INTRODUCTION}

Transportation of timber products from harvesting sites to conversion facilities is the largest cost component of timber harvesting operations. Forest transportation planning problems (FTPPs) have traditionally involved finding routes that minimize log hauling and road construction costs. ${ }^{[1,2]}$ FTPPs that consider both variable (log hauling) and fixed costs (road construction) are a special case of the fixed charge transportation problem (FCTP), which is known as a NP-hard combinatorial optimization problem. ${ }^{[3,4]}$ Mixed-Integer Programming (MIP) has been used to optimally solve FCTP but its application is limited to small- and medium-scale problems because solution time grows exponentially with problem size. ${ }^{[4,5]}$ Large-scale FTPPs have been solved using several heuristic approaches. Although these approximation algorithms do not guarantee optimality, they can effi-

\footnotetext{
* Correspondence: Pengpeng Lin; Email: linp@uwstout.edu; Address: Department of Mathematics, Statistics and Computer Science, University of Wisconsin Stout, Menomonie WI, 54751, United States.
} 
ciently provide high-quality solutions for large and complex problems in a practical amount of time. ${ }^{[6-8]}$ Examples of approximation approaches used to solve large-scale FTPPs with fixed and variable costs include MINCOST, ${ }^{[9]}$ NETCOST, ${ }^{[10]}$ NETWORK 2000[11] and NETWORK 2001. [12] Although these approaches have been widely used, their formulations are set to minimize total transportation costs only and cannot consider side constraints based on additional attributes of road segments. Increasing environmental concerns related to the transport of timber products have introduced negative impacts such as increased erosion from road surfaces and its subsequent impact of water quality into FTPPs. ${ }^{[13]}$ These environmental considerations and requirements introduce constraints making FTPPs more complex than the traditional cost minimization problems. A few studies have incorporated environmental impacts into FTPPs by assigning an environmental cost to potential sediment yields from forest roads and used NETWORK 2000 to find least-cost routes. ${ }^{[14,15]}$ However, it is difficult and arbitrary to assign an economic value to a negative environmental impact.

Land managers are often asked to address road system issues at a large landscape scale across multiple time periods. To address this research need, we developed an approach (ACOLS) combining the ant colony optimization ${ }^{[16]}$ and a local search method for solving real-world, large-scale, multiperiod FTPP considering fixed and variable costs and sediment constraints. Three major routines were incorporated into the ACOLS to ensure solution quality and efficiency. First, the ACOLS simultaneously finds least-cost routes from all timber sales to the final destination to allow sharing road segments among individual routes from timber sales to avoid creating unnecessary circuits between any two nodes in the transportation network, which reduces fixed costs and sediment yields. Second, a two-stage process was implemented to increase the probability of finding feasible and high-quality solutions efficiently. During stage I, the algorithm only considers sediment yield information on each road segment to quickly find feasible solutions. During stage II, sediment and cost information per road segment are considered to select feasible solutions resulting in least-cost routes. Third, a 1opt local search refinement procedure was implemented to improve solution quality. To evaluate the applicability of the developed approach, we initially applied the ACOLS to a hypothetical FTPP. Then, the ACOLS was applied to 10 FTPP instances created using the same FTPP. Finally, we applied our ACO algorithm to solve a real-world, large-scale FTPP with multi-periods including thousands of road segments, hundreds of timber sales locations, ten planning periods and multiple destination mills. The experimental results show that ACOLS was able to find feasible solutions for all test cases.

\section{MIP MODEL FORMULATION}

As mixed-integer programming can solve moderate-size problems with reasonable times, it was used on the hypothetical problem instances in the experiments to compare with the ACOLS performance. Moreover, the FTPPs with multiple planning periods, which is studied in this work, can be well presented using the mixed-integer programming formulation:

$$
\begin{aligned}
& \text { Minimize: } \\
& \qquad \begin{aligned}
f(P, G, E)= & \sum_{p \in P} \sum_{g \in G} \sum_{i j \in E}\left(V C_{i j, p} \times v l_{i j, g, p} \times B_{i j, p}\right) \\
& +\left(F C_{i j, p} \times I_{i j, p}\right)
\end{aligned}
\end{aligned}
$$

Subject to:

$$
\begin{aligned}
& \sum_{i j \in E}\left(\operatorname{sed}_{i j, p} \times B_{i j, p}\right) \leq \text { SedRct }_{p} \quad \forall p \in P \\
& \operatorname{vol}_{j, g, p}+\sum_{i j \in L_{j}} \operatorname{vol}_{i j, g, p}-\sum_{j i \in L_{j}} \operatorname{vol}_{j i, g, p}=0 \\
& \forall j \in S, \forall g \in G, \forall p \in P \\
& \sum_{i j \in L_{j}} \operatorname{vol}_{i j, g, p}-\sum_{j i \in L_{j}} \operatorname{vol}_{j i, g, p}=0 \\
& \forall j \in T, \forall g \in G, \forall p \in P \\
& \sum s \in \operatorname{Svol}_{s, g, p}-\sum i j \in L_{j} \operatorname{vol}_{i j, g, p}=0 \\
& \forall j \in D, \forall g \in G, \forall p \in P \\
& M \times B_{i j, p}-\left(\operatorname{vol}_{i j, g, p}+\operatorname{vol}_{j i, g, p}\right) \geq 0 \\
& \forall i j \in E, \forall g \in G, \forall p \in P \\
& \sum_{p \in P} B_{i j, p}-I_{i j, p} \geq 0 \quad \forall i j \in E \\
& \text { vol }_{i j, g, p} \geq 0 \quad \forall i j \in E, \forall g \in E, \forall p \in P \\
& B_{i j, p}, I_{i j, p} \in[0,1] \quad \forall i j \in E, \forall p \in P
\end{aligned}
$$

\section{where the variables are defined as:}

- $v_{i j, g, p}=$ timber volume of product $g\left(\mathrm{~m}^{3}\right)$ transported over the edge $i j$ (edge having vertex $i$ as the from-vertex and $j$ as the to-vertex) during period $p$.

- $V C_{i j, p}=$ variable cost per unit of volume $\left(/ \mathrm{m}^{3}\right)$ for timber transported over edge $i j$ during period $p$.

- $F C_{i j, p}=$ fixed cost $(\$)$ for timber transported over 
edge $i j$ during period $p$.

- $\operatorname{sed}_{i j, p}=$ amount of sediment (tons) expected to erode from edge $i j$ during period $p$ due to the traffic of heavy log-trucks.

- $v o l S_{s, g, p}=$ timber volume $\left(\mathrm{m}^{3}\right)$ of product $g$ required to be transported from timber sale $s$ during period $p$.

- SedRct $_{p}=$ maximum amount of sediment allowed for the entire transportation network during period $p$.

- $B_{i j, p}=1$ if there is timber volume of any product transported over edge $i j$ during period $p ; 0$ otherwise.

- $I_{i j, p}=1$ if there is timber volume of any product transported over the $i j$ edge during period $p$ for the first time in the planning horizon; 0 otherwise.

\section{and the symbols are defined as:}

- $E=$ number of edges in the transportation network.

- $V=$ number of vertices in the transportation network.

- $L_{j}=$ set of edges having vertex $j$ as a from-or tovertex.

- $S=$ set of vertices representing timber sale locations.

- $D=$ set of vertices representing mill destinations.

- $T=$ set of intermediate vertices (representing neither timber sales nor mills).

- $P=$ number of time period in the planning horizon.

- $G=$ number of timber products.

- $M=$ constant equal or greater than the total timber volume transported over the planning horizon.

Equation 1 is the objective function to minimize total transportation cost over the planning horizon. The first constraint set (Equation 2) limits the total amount of sediment (tons) expected to be produced from the entire transportation network in each planning period by accounting for sediment yield on each edge $\left(s e d_{i j, p}\right)$ if traffic of any product exists in period $p$. Equations 3-5 are conservation of flow constraints that ensure all timber volume entering the transportation network during each period is routed through the network to the destination mills. Constraint set (Equation 3) ensures that timber volume enters the network through timber sales $S$ and the flow will not go back. Constraint set (Equation 4) ensures that the sum of the volume of each product entering a vertex $j$ must equal to the sum of the volume leaving that vertex. Constraint set (Equation 5) ensures that the timber volume must be transported to the designated mill destinations $D$. Constraint set (Equation 6) represents road trigger constraints to ensure that if there is timber volume of any product transported over edge $i j$, in either direction, sediment amount is accounted. The Equation 6 restricts that the traffic can only go in one direction over any edge $i j$ thus preventing the circler routes. Constraint set (Equation 7) also represents road trigger constraints to ensure that if there is sediment accounted over edge $i j$ (thus timber volume traffic exists in either direction) during one or more periods, then the fixed cost for the edge $i j$ is accounted only the first period traffic exists. Constraints (Equations 8 and 9) are non-negativity and binary value constraints of the formulation.

The MIP formulation illustrates that the dimensionality of the FTPP increases as a function of the size of the transportation network (vertices and edges), number of timber products, and planning periods. Total number of variables and constraints in the formulation:

- Total number of variables $=2 \times E \times P \times(G+1)$

- Continuous $=2 \times E \times P \times G$

- Binary $=2 \times E \times P$

- Total number of constraints $=4 \times(E \times P)+(V \times$ $P \times G)+P$

- Sediment $=P$

- Conservation of flow $=(V \times P \times G)$

- Sediment road trigger $=(E \times P)$

- Fixed cost road trigger $=(E \times P)$

- Non-negativity $=(E \times P)$

- Binary value $=(E \times P)$

\section{ACOLS}

The ACOLS developed in this work follows the general ACO framework ${ }^{[16,17]}$ and is specifically designed to solve constrained FTPP considering fixed and variable costs. Customized routines were introduced in the solution finding procedure for the algorithm performance and solution quality. A description of the ant traveling mechanism and solution building process, 1-opt local search solution refinement, pheromone update, stopping criterion, and a summary of the optimization process are presented.

\subsection{Ant traveling mechanism}

A finite number of ants, one placed in each origin vertex (timber sale location), search for least-cost routes from each origin vertex to the selected destination vertex (mill location) in the transportation network. Starting with planning period one, the first ant is randomly located at an origin vertex, and finds the least-cost route from the origin to destination vertex by moving sequentially through adjacent vertices. The second ant is then randomly located in a different origin vertex to find the origin-destination route. Once all ants have found a route connecting each origin vertex to its selected destination vertex for the first planning period, ants start finding origin-destination routes for the second planning period and so on. An iteration is completed when origin-destination routes have been found for all periods. 
To focus the route construction process in the feasible solution space and improve solution quality, two stages were implemented into the ACOLS. During stage I, ants are set to rapidly find feasible solutions without consideration of transportation costs. Stage II is set to start after the first feasible solution is found to ensure both solution feasibility and quality. Ants select what vertex to visit next based on a transition probability computed for each adjacent edge, which is calculated differently for each stage. During stage I, the transition probability is calculated as follows:

$$
\text { Stage I } \rightarrow P_{i j}(t)=\frac{\left(\tau_{i j}\right)^{\alpha} \times\left(\operatorname{Sed}_{i j}^{-1}\right)^{\beta}}{\sum_{i k \in N_{i}}\left(\tau_{i k}\right)^{\alpha} \times\left(\operatorname{Sed}_{i k}^{-1}\right)^{\beta}}
$$

where $P_{i j}(t)$ is the transition probability with which an ant selects edge $i j$ during iteration $t, N_{i}$ is the set of edges having vertex $i$ as the from-vertex, $\alpha$ and $\beta$ are parameters that control the relative importance of the pheromone trail intensity $\left(\tau_{i j}\right)$ and a heuristic value that indicates the desirability of selecting edge $i j$. During state I, it is calculated as the reciprocal of the associated sediment amount on the edge $\left(\mathrm{Sed}_{i j}^{-1}\right)$. During stage II, the transition probability incorporates the reciprocal of all three attributes associated to each edge: fixed cost (converted into a per unit basis), variable cost, and sediment amount (Equation 11).

Stage $\mathrm{II} \rightarrow P_{i j}(t)=$

$\frac{\left(\tau_{i j}\right)^{\alpha} \times\left[\lambda\left(\frac{F C_{i j}}{v o l T_{i j}}+V C_{i j}\right)^{-1}+(1-\lambda)\left(\operatorname{Sed}_{i j}^{-1}\right)\right]^{\beta}}{\sum_{i k \in N_{i}}\left(\tau_{i k}\right)^{\alpha} \times\left[\lambda\left(\frac{F C_{i k}}{v o l T_{i k}}+V C_{i k}\right)^{-1}+(1-\lambda)\left(\operatorname{Sed}_{i k}^{-1}\right)\right]^{\beta}}$

where $F C_{i j}$ is the total fixed cost on edge $i j, v o l T_{i j}$ the total timber volume traveled through edge $i j, \lambda$ the weight given to costs per unit of volume, $(1-\lambda)$ the weight given to sediment amount, and $R$ the set of origin vertices selecting edge $i j$ as part of their least-cost route during the previous iteration.

While constructing a route, if an ant visits a vertex that is part of a previously constructed origin-destination route with the same destination vertex, the ant stops and the remaining of the constructed route is attached to the route being constructed. Ants are also restricted from visiting previously visited vertices to avoid forming infinite cycles. When an ant is at vertex $v$ and all adjacent vertices have been previously visited by the ant, the ant moves back to the previous vertex and marks vertex $v$ as unavailable. This back-tracking process continues until vertices become available. This ant travel mechanism is designed specifically for the FTPP with fixed and variable costs because sharing road segments among multiple timber sales is considered desirable from both economic and environmental perspectives.

\subsection{Local search solution refinement}

Local search procedures have shown to improve solution quality for different ACO based algorithms. ${ }^{[18,19]}$ We implemented a local search in the form of a 1-opt routine into our algorithm, which looks at each vertex and its adjacent vertices along individual origin-destination routes to find potential shortcuts that might improve solution quality. Similar to the calculation of transition probabilities (Equations 10 and 11), solution quality evaluated in the 1-opt routine is based only on sediment amount during stage I and based on all three edge attributes during stage II. For a given vertex $v_{i}$ forming part of an origin-destination route $\left(v_{\text {or } i} \rightarrow \cdots \rightarrow v_{i} \rightarrow \cdots \rightarrow v_{\text {dest }}\right)$, the local search routine looks at adjacent vertices of $v_{i}$ along the route and evaluates if eliminating $v_{i}$ from the route and alternatively connecting $v_{i-1}$ and $v_{i+1}$ results in lower costs and sediment amount.

\subsection{Pheromone update}

Pheromone evaporation is a procedure to avoid a too rapid convergence towards suboptimal solutions and allows the exploration of other areas of the solution space. In the ACOLS, pheromone trail intensity is updated as follows:

$$
\tau_{i j}(t+1)=\tau_{i j}(t) \times \rho+\Delta \tau_{i j}
$$

The first component is the current pheromone trail intensity on edge $i j$ during iteration $t$, which is multiplied by $0 \leq \rho \leq 1$, where $(1-\rho)$ represents the pheromone evaporation rate between iterations $t$ and $t+1$. The second component is the newly added pheromone amount to edge $i j$ and is calculated consistently with the purpose of obtaining feasible solutions during stage I (Equation 13), and feasible and high-quality solutions during stage II (Equation 14).

$$
\text { Stage I } \rightarrow \Delta \tau_{i j}= \begin{cases}\frac{Q}{S e d_{i j}} & \text { if edge } i j \text { is part of the solution } \\ 0 & \text { otherwise }\end{cases}
$$




$$
\text { Stage II } \rightarrow \Delta \tau_{i j}=\left\{\begin{array}{l}
\lambda \frac{Q}{\left(\frac{F C_{i j}}{v o l T_{i j}}+V C_{i j}\right)}+(1-\lambda) \frac{Q}{\operatorname{Sed}_{i j}} \\
0
\end{array}\right.
$$

where $Q$ is a constant set to ensure that the amount of pheromone added to edge $i j$ slightly increases its selection probability during the next iteration. Based on previous trial runs, $Q$ was 0.00001 in the experiments.

\subsection{Stopping criterion}

Three stopping criteria were implemented into the ACOLS to address stagnation of solution quality and improve computing time. During stage I, the algorithm tracks the number of consecutive solutions generated, and if a user-defined maximum number of iterations ( $\left.I t_{s e d}\right)$ is exceeded without finding a feasible solution, the algorithm stops and reports "no feasible solution found". During stage II, the algorithm also tracks the number of consecutive infeasible solutions, and if it exceeds a user-defined maximum number $\left(I t_{\text {infeas }}\right)$, the algorithm stops and reports the best feasible solution found. Each time a feasible solution is found, the algorithm resets the counter to zero. Moreover, the number of consecutive unimproved solutions is tracked during stage II, and if it exceeds a user-defined maximum number $\left(I t_{\text {feas }}\right)$, the algorithm stops and reports the best feasible solution found so far. For our applications presented in this study, $I t_{\text {sed }}$, $I t_{\text {infeas }}$, and $I t_{\text {feas }}$ were all set to 10,000 .

\subsection{Optimization process}

At the end of each iteration, the 1-opt local search routine is applied to each origin-destination route in each planning period. The objective function is then computed and the solution feasibility is evaluated. As aforementioned, at the beginning of the optimization process during stage I, solution construction, local search, and solution evaluation are based on sediment amounts associated to every edge in the transportation network. If the current solution is better (i.e. lower total sediment amount) than the best solution found so far, the best solution is replaced by the current solution, and the pheromone is updated also based on the sediment amount (Equation 13). Stage II starts as soon as a feasible solution is found, and in this case, solution construction, local search, and solution evaluation are based on all three attributes (i.e., fixed cost, variable cost, and sediment amount) associated to every edge. Similar to stage I, when the current solution is better (i.e., lower total transportation cost) than the best solution found so far, the best solution is replaced, and the pheromone is updated also based on the three edge attributes (Equation 14). During both stages, when the current solution is either infeasible or worse than the best solution found so

Published by Sciedu Press if edge $i j$ is part of the solution

otherwise far, pheromone update is not performed and a new iteration starts. The ACOLS continues generating alternative solutions in each iteration until one of the three stopping criteria is reached, at which point the best solution is reported.

\section{Algorithm application}

Similar to most heuristic optimization techniques, the parameters of the ACO based algorithms have a significant effect on algorithm performance and fine tuning their values is required to ensure high-quality solutions. ${ }^{[20]}$ We conducted a full factorial search to find the best parameter values using the single-period, 500-edge hypothetical FTPP that was used in. ${ }^{[21]}$ After finding the best parameter value for the hypothetical FTPP, the ACOLS was applied to ten different problem instances ${ }^{[22]}$ to test the robustness in terms of its ability to consistently find high-quality solutions on different FTPP of similar size using the same parameter values found for the original hypothetical problem. Moreover, the ACOLS was applied to a large-scale, real-world FTPP with multiple planning periods to illustrate its application under more realistic settings.

\subsection{Parameter setting and hypothetical FTPPs}

In the ACOLS, four main parameters influence its performance: the importance of the amount of pheromone amount $(\alpha)$, the importance of the three edge attributes $(\beta)$, pheromone persistence rate $(\rho)$, and the relative importance of both costs over the sediment amount $(\lambda)$. A range from 0 to 1 was partitioned into 21 discrete values at a pace of 0.05 for $\alpha, \beta$ and $\rho$, and into 11 discrete values at a 0.1 pace for $\lambda$ resulting in a total of 101,871 parameter combinations. The ACOLS was run 10 times for each parameter combination and the combination producing the highest average solution quality was selected as the best parameter combination.

As aforementioned, the hypothetical FTPP considers a road transportation network formed by 500 road segments where traffic is allowed in both directions (thus 1,000 edges) and 200 vertices. A total volume of $36,500 \mathrm{~m}^{3}$ is to be delivered from 25 timber sale locations to one mill destination in a single planning period. Timber volume per sale location varied from $1,029 \mathrm{~m}^{3}$ to $1,905 \mathrm{~m}^{3}$. Variable cost, fixed cost, and sediment yield per edge ranged from $\$ 0.01 / \mathrm{m}^{3}$ to $\$ 10 / \mathrm{m}^{3}$, from $\$ 0$ to $\$ 23,000$ for road construction and maintenance, and from 0.4 to 200 tons, respectively. Based on our MIP formulation (Equations 1-9), this medium-scale problem has 
2,000 variables and 2,201 constraints. We also considered the four cases with increasing level of sediment constraint. Case I is a cost minimization problem without sediment constraint, cases II and III are cost minimization problems subject to increasing levels of upper-bound sediment constraints, and case IV is a sediment minimization problem without cost consideration. The results of cases I and IV provided upper and lower bounds of sediment yields from the road network, and thus were used in determining appropriate sediment constraint values for cases II and III. The parameter search process described above was conducted on the hypothetical FTPP for each case to determine if parameter values were also sensitive to the sediment restriction level.

Moreover, we created a set of ten problem instances on the same hypothetical road transportation network to test the robustness of the algorithm performance using the same parameter values found during the parameter search process. These problem instances were created by randomly changing the location of the 25 timber sales (origin vertices) and the single mill destination in the network. Timber volume of sale locations and attributes (fixed and variable costs and sediment amount) on each edge remained the same for all problem instances. For each problem instance, we also considered the four cases. As aforementioned, cases I and IV were solved to obtain lower and upper limits of sediment constraint values for cases II and III, which were set at one third and two thirds of the difference between the upper and lower limits subtracted from the upper limit. Consequently, the level of sediment constraint increases equally from $0 \%$ (case I) to $33 \%$ (case II), to $66 \%$ (case III), and to $100 \%$ (case IV).

The ACOLS, as any heuristic optimization algorithms, do not guarantee solution optimality. Therefore, the best solution for each case found by the ACOLS using the best parameter values were compared with optimal solutions found by a commercially available MIP solver. We used CPLEX version $12.5^{[23]}$ with default optimization setting parameters to solve all problems to optimality on a desktop computer with four dual processors at $3.07 \mathrm{GHz}$ and $3.00 \mathrm{~GB}$ of RAM.

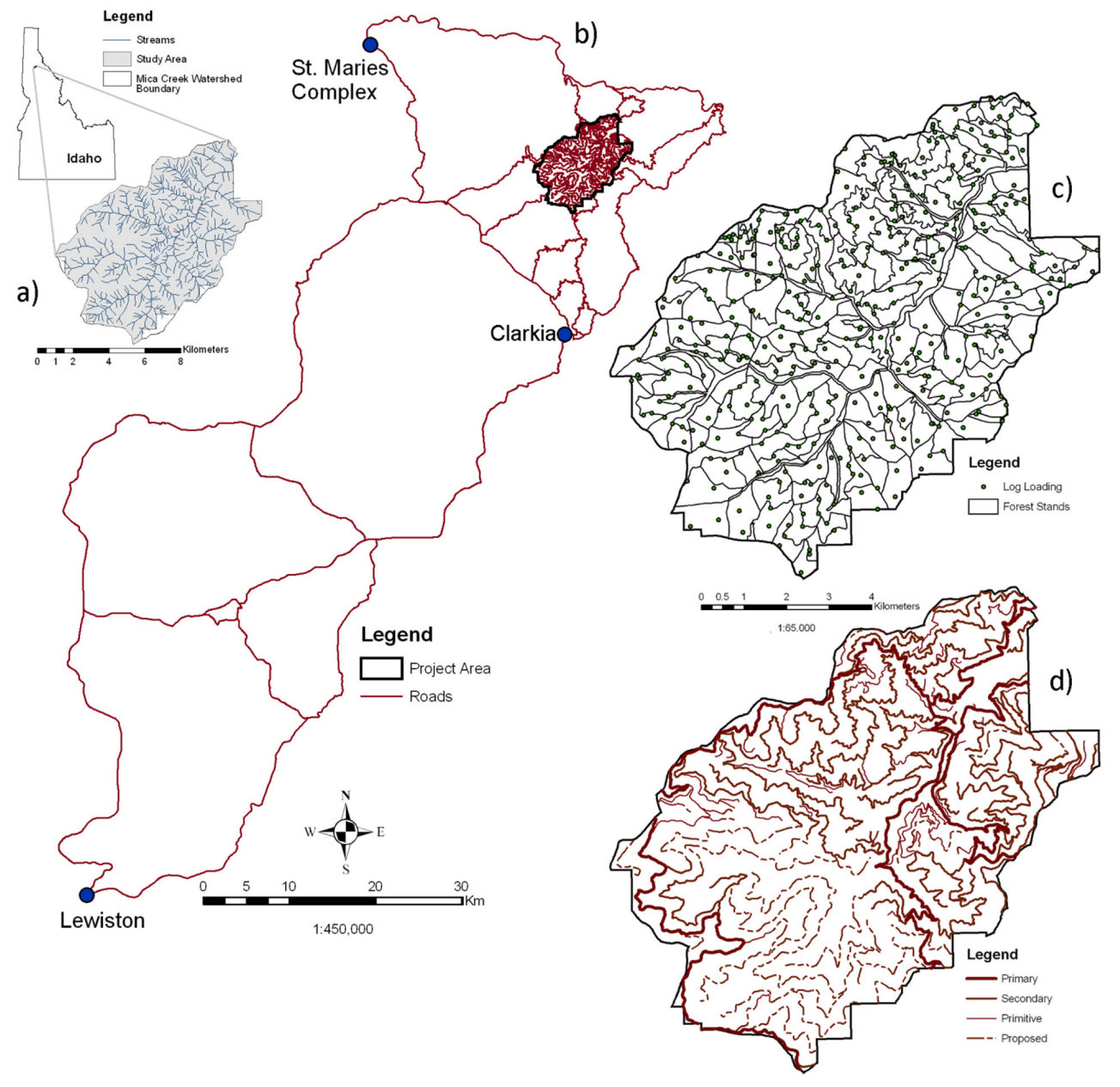

Figure 1. Study area located in the upper part of Mica Creek watershed in Northern Idaho, USA 


\subsection{Real-world large-scale FTPP}

The ACOLS was also applied to solve a real-world, largescale FTTP considering multiple products, mill destinations, planning periods, hundreds of timber sale locations, and a road network formed by thousands of edges and vertices. The study area is located in the southern portion of the Mica Creek watershed, part of the St. Joe River basin in northern Idaho, USA (see Figure 1a). The study area ${ }^{[14]}$ is owned and managed by Potlatch Forest Holdings, Inc. (Potlatch). In the study area of 7,070 ha, there were 261 harvest units ranging from 1.4 to 183.3 ha in size inside which 407 log landing locations were predetermined for timber harvest according to harvesting systems (ground-based vs. cable logging) based on average slope within harvest units (see Figure 1c). Total length of the road transportation network inside the study area is $342 \mathrm{~km}$ (see Figure 1d); $271 \mathrm{~km}$ are existing roads and $71 \mathrm{~km}$ are proposed roads for future access to harvest units. Existing roads were classified by Potlatch into primary, secondary, and primitive roads based on road standards such as width, maximum grades, and design vehicle. The harvest schedule provided by Potlatch consisted of expected harvestable timber volume by product in each harvest unit, year of harvest, and destination mill. The harvest schedule included six 5-year planning periods $(7,12, \ldots, 27)$ for the next 27 years and four 10-year planning periods afterward up to 75 years $(35, \ldots, 75)$. Total harvestable volume from the 261 harvest units over the 75 year planning horizon is approximately $422,000 \mathrm{~m}^{3}$ (see Table 1), which was separated into two products: logs and pulp. St. Maries and Lewiston in Idaho, located about $45 \mathrm{~km}$ and $100 \mathrm{~km}$ away from the study area were selected as the product destinations for logs and pulp respectively (see Figure 1b). An interest rate of 5\% was considered to calculate discounted transportation costs.

Table 1. Total harvest volume $\left(\mathrm{m}^{3}\right)$ scheduled to be delivered by period

\begin{tabular}{lll}
\hline Period & Years from present & Harvestable volume \\
\hline 1 & 7 & $70,096.7$ \\
2 & 12 & $18,967.0$ \\
3 & 17 & $140,099.8$ \\
4 & 22 & $17,808.7$ \\
5 & 27 & $53,630.7$ \\
6 & 35 & $41,828.3$ \\
7 & 45 & $5,656.7$ \\
8 & 55 & $28,022.9$ \\
9 & 65 & $25,513.3$ \\
10 & 75 & $20,515.6$ \\
Total & & $422,139.7$ \\
\hline
\end{tabular}

The ACOLS was applied to this real-world, large-scale FTPP considering the four cases mentioned above. Case I minimizes transportation costs for all ten planning periods with- out a sediment constraint, and case IV minimizes sediment amount from the entire road network for all periods. The sediment amount by period resulting from the best solution found by the ACOLS for case I and the sediment amount by period from case IV provided the upper and lower limits of the sediment constraint values for cases II and III. Similar to the ten problem instances, the sediment constraint level for each period is set to increase from $0 \%$ (case I) to $33 \%$ (case II), to $66 \%$ (case III), and to $100 \%$ (case IV).

\section{RESULTS AND DISCUSSION}

Using the best parameter combination found by the search, the ACOLS was able to find near-optimal solutions for all test cases of the original hypothetical FTPP and for all cases in the ten problem instances. Moreover, it was able to find feasible solutions for all cases of the real-world, large-scale problem. Results of the parameter search process, objective function values, and transportation routes of all applications are presented below.

Table 2. Best combinations of parameter values found by the full factorial parameter search process for all cases of the original hypothetical FTPP

\begin{tabular}{lllll}
\hline Case & $\alpha$ & $\beta$ & $\rho$ & $\lambda$ \\
\hline I & 0.50 & 0.4 & 0.55 & 1.0 \\
II & 0.50 & 0.9 & 0.60 & 0.7 \\
III & 0.50 & 0.7 & 0.65 & 0.7 \\
IV & 0.45 & 1.0 & 0.15 & 0.0 \\
\hline
\end{tabular}

\subsection{Parameter setting and hypothetical FTPPs}

The parameter search process performed on the original hypothetical FTPP resulted in a different combination of parameter values for each of the four cases (see Table 2). This indicates that fine-tuning of parameter values is necessary to achieve high-quality solutions not only for different applications but even when constraint levels change in the same application. Although previous studies have shown that best parameter values are application specific, ${ }^{[2,25]}$ to our knowledge, no studies have examined the relationship between parameter values and constraint level. Commonly, one parameter value combination is applied for a given application regardless of constraint level.

Some best parameter values remained relatively similar among the four cases while others varied significantly. For example, the range of $\alpha$ remained between 0.45 (cases I-III) and 0.5 (case IV) across the four cases, whereas $\beta$ presented a large variation among cases and did not seem to correlate with sediment constraint level. However, when sediment is considered (cases II-IV), $\beta$ values became larger than $\alpha$ values, which indicates that more importance is given to 
the edge attributes (sediment being one of them) than to the amount of pheromone on the edges. The parameter controlling pheromone persistence $(\rho)$ also widely varied among the cases. The largest $\rho$ values of $0.6-0.65$ were selected for the constrained cases (II and III) where the three edge attributes are considered. For case I, which includes only two edge attributes (fixed and variable costs), the value of $\rho$ decreased slightly to 0.55 and it was the lowest $(0.15)$ for case IV, which considers only one edge attribute, sediment amount. These results might indicate higher persistence of pheromone is required when more attributes are considered. Lastly, parameter $\lambda$ presents the largest variability but it directly correlates with the constraint level. As expected, all weight (i.e., $\lambda=1.0$ ) is given to the costs associated with each edge in the road network when the objective is to minimize total transportation costs. For the sediment constrained cost minimization cases (II and III) $70 \%$ importance is given to costs on the edges, and all weight is given to the sediment amount on the edges for the sediment minimization case (IV).

Table 3. Comparison of objective function values between MIP and ACO solutions for the four cases of the original hypothetical FTPP

\begin{tabular}{lllll}
\hline Case & ACOLS (Objective value) & Sediment constraint value (tons) & MIP (Objective value) & Percent difference \\
\hline I & $1,496,562$ & N/A & $1,496,562$ & $0.00 \%$ \\
II & $1,637,860$ & 2,000 & $1,585,393$ & $3.31 \%$ \\
III & $2,086,280$ & 1,500 & $2,008,344$ & $3.88 \%$ \\
IV & 948.6 & N/A & 948.6 & $0.00 \%$ \\
\hline
\end{tabular}

The ACOLS solutions for the four cases of the original hypothetical FTPP reported in Table 3 are those obtained from using the best parameter values found by the exhaustive search process. CPLEX was able to find optimal solutions for all cases within a reasonable amount of time. It took approximately 1 minute for case I, 1.2 hours for case II, 54 hours for case III, and 48 minutes for case IV. The ACOLS was able to match the optimal MIP solution found by CPLEX for cases I and IV and found near-optimal solutions for the constrained cases with optimality level of $96.70 \%$ and $96.12 \%$ for cases II and III, respectively.

Table 4. Objective function value comparisons between MIP and ACO solutions for cases II and III of the ten FTPP instances

\begin{tabular}{|c|c|c|c|c|c|}
\hline Instance & Case & $\begin{array}{l}\text { ACO objective function } \\
\text { value (\$) }\end{array}$ & Sediment constraint (tons) & $\begin{array}{l}\text { MIP objective function } \\
\text { value (\$) }\end{array}$ & Percent difference \\
\hline \multirow[t]{2}{*}{1} & II & 887,719 & 2,159 & 878,749 & 1.02 \\
\hline & III & $1,027,550$ & 1,727 & 981,203 & 4.72 \\
\hline \multirow[t]{2}{*}{2} & II & $1,416,090$ & 2,490 & $1,415,960$ & 0.01 \\
\hline & III & $1,619,740$ & 1,860 & $1,563,669$ & 3.59 \\
\hline \multirow[t]{2}{*}{3} & II & $1,055,330$ & 2,254 & $1,048,768$ & 0.63 \\
\hline & III & $1,174,630$ & 1,746 & $1,170,956$ & 0.31 \\
\hline \multirow[t]{2}{*}{4} & II & 914,972 & 2,449 & 910,152 & 0.53 \\
\hline & III & $1,043,763$ & 1,778 & $1,043,763$ & 0.00 \\
\hline \multirow[t]{2}{*}{5} & II & $1,203,500$ & 2,445 & $1,181,284$ & 1.88 \\
\hline & III & $1,301,920$ & 1,945 & $1,260,541$ & 3.28 \\
\hline \multirow[t]{2}{*}{6} & II & $1,212,620$ & 2,354 & $1,208,610$ & 0.33 \\
\hline & III & $1,398,950$ & 1,760 & $1,355,860$ & 3.18 \\
\hline \multirow[t]{2}{*}{7} & II & $1,089,140$ & 2,672 & $1,066,148$ & 2.16 \\
\hline & III & $1,164,660$ & 1,978 & $1,164,368$ & 0.03 \\
\hline \multirow[t]{2}{*}{8} & II & $1,241,760$ & 2,660 & $1,229,392$ & 1.01 \\
\hline & III & $1,418,220$ & 1,971 & $1,361,841$ & 4.14 \\
\hline \multirow[t]{2}{*}{9} & II & $1,410,850$ & 2,262 & $1,378,432$ & 2.35 \\
\hline & III & $1,679,540$ & 1,734 & $1,636,147$ & 2.65 \\
\hline \multirow{2}{*}{10} & II & $1,403,150$ & 2,342 & $1,394,355$ & 0.63 \\
\hline & III & $1,634,760$ & 1,750 & $1,628,223$ & 0.40 \\
\hline \multirow{2}{*}{ Average } & II & & & & 1.06 \\
\hline & III & & & & 2.23 \\
\hline Total average & & & & & 1.64 \\
\hline
\end{tabular}


Table 5. Comparison of computing times (sec) for a single run of the ACO algorithm and the MIP solver for the constrained cases of problem instances

\begin{tabular}{llllll}
\hline \multirow{2}{*}{ Instance } & \multicolumn{2}{l}{ ACOLS } & & & MIP \\
\cline { 2 - 3 } \cline { 6 - 6 } & Case II & Case III & & Case II & Case III \\
\hline 1 & 434 & 363 & & 3,254 & 31,722 \\
2 & 263 & 2,732 & & 62,314 & 90,973 \\
3 & 790 & 428 & & 3,532 & 21,893 \\
4 & 708 & 396 & & 4,732 & 36,385 \\
5 & 190 & 29,051 & & 3,110 & 149,585 \\
6 & 304 & 8,885 & & 4,344 & 97,582 \\
7 & 905 & 371 & & 1,333 & 31,540 \\
8 & 371 & 10,722 & & 8,458 & 55,516 \\
9 & 509 & 332 & & 1,182 & 152,629 \\
10 & 962 & 419 & & 7,225 & 59,597 \\
Average & 544 & 5,370 & & 9,948 & 72,742 \\
\hline
\end{tabular}

Results from applying the ACOLS to the ten different problem instances also show evidence of good algorithm performance by consistently providing high-quality solutions. The ACOLS was able to match seven out of ten optimal MIP solutions for case I problems and found near-optimal solutions for the remaining three instances $(99.9 \%, 98.25 \%$, and $99.74 \%$ optimal for instances 1, 2, and 7, respectively). For case IV, the ACOLS matched optimal MIP solutions for all but one problem instance (problem instance 2), which was 99.63\% optimal. For the constraints cases, ACOLS solutions averaged $98.36 \%$ optimality (see Table 4 ). ACOLS solution quality was slightly better for case II problems compared to case III because of the more relaxed constraint level. Solution quality ranged from $97.84 \%$ to $99.99 \%$ optimal for case II problems and from $95.28 \%$ to $100 \%$ optimal for case III problems. This is expected because as constraint becomes stricter fewer feasible solutions exist and the algorithm spends more time evaluating a larger number of infeasible solutions.

Although the ACOLS did not match optimal MIP solutions for all cases, it was able to find near-optimal solutions for all constrained hypothetical FTPP in a fraction of the computing time (see Table 5). Time required by the ACOLS and the CPLEX to solve case II and case III problems among instances varied from $190 \mathrm{~s}$ to $962 \mathrm{~s}$, from $332 \mathrm{~s}$ to $29,051 \mathrm{~s}$, from $1,182 \mathrm{~s}$ to $62,314 \mathrm{~s}$, and from $21,893 \mathrm{~s}$ to 152,629 s. In average, ACOLS solutions were obtained within $5.5 \%$ and $13 \%$ of the times required to find optimal solutions using MIP for case II and case III problems, respectively. Due to the increased complexity of the case III problems, ACOLS and MIP solution times increased about 10 and 7.5 times, respectively, from those of case II problems.

Table 6. Best ACO solution for the four cases of the real-world, large-scale FTPP

\begin{tabular}{lllll}
\hline & Case I & Case II & Case III & Case IV \\
\hline Total cost $(\$)$ & $6,184,763$ & $6,341,404$ & $6,721,262$ & $9,007,215$ \\
Variable cost $(\$)$ & $5,587,879$ & $5,752,740$ & $6,086,440$ & $8,359,508$ \\
Fixed cost $(\$)$ & 596,884 & 588,664 & 634,822 & 647,707 \\
Total sediment (ton) & 22,791 & 18,376 & 16,636 & 14,722 \\
Total road length $(\mathrm{km})$ & 285.2 & 287.6 & 285.8 & 286.5 \\
\hline
\end{tabular}

\subsection{Real world large-scale FTPP}

After applying the ACOLS to solve the real-world, largescale FTPP, feasible solutions were found for all four cases. Total transportation cost increased about $46 \%$ from about $\$ 6.2 \mathrm{M}$ in case I (cost minimization) to $\$ 9.00 \mathrm{M}$ in case IV (sediment minimization) (see Table 6). Sediment amount decreased about $35 \%$ from case I to case IV $(22,791$ tons vs. 14,722 tons). For case II, which restricted sediment by about $19.4 \%$ from total sediment amount of the unrestricted case I (18,376 tons), transportation cost increased $2.5 \%$. For case III, where sediment was restricted to about $27.0 \%$ of the unrestricted case I (16,636 tons), transportation cost rose $8.7 \%$. These results likely indicate that sediment restriction level is not proportional to transportation cost increment. Restricting total sediment amount by a given percent results in a smaller percent increase in costs. Total road length inside the study area remained relatively similar, ranging from 285.2 to
$287.6 \mathrm{~km}$, among solutions for all cases. This is likely because sediment amount is a function of several road design factors (road gradient, cut and fill slopes, rock fragment percent on road surface, etc.) not just length. Thus, as sediment restriction level increases, road segment producing less sediment, not necessarily shorter, are selected. Solution time varied from $79 \mathrm{hr}$. to $91 \mathrm{hr}$. and no clear correlation between sediment restriction level and solution time was observed from a single ACOLS run. This might be explained by dynamic interactions among the three stopping criteria and ACOLS's probabilistic nature.

As aforementioned, sediment restriction per period were set based on the sediment amount associated to the best found solution for case I and objective function value by period for case IV. Table 7 shows transportation cost, associated sediment amounts, and sediment restriction values (for cases II and III) by period for the best ACOLS solutions of the four 
cases. As expected, the same pattern of increasing costs and decreasing sediment amount from case I through case IV can be observed for each period. For all four cases, transportation cost for periods one and two are approximately $73 \%$ of the total transportation costs. This may be because proposed roads need to be constructed to access a large number of timber sales and transport large timber volume scheduled in the early periods (see Table 1), which increases both fixed costs and variable costs.

Table 7. Transportation cost and sediment amount by period associated to the best solution found by the ACO algorithm for the four cases of the real-world, large-scale FTPP

\begin{tabular}{|c|c|c|c|c|c|c|c|c|c|c|}
\hline \multirow[b]{2}{*}{ Period } & \multicolumn{2}{|l|}{ Case I } & \multicolumn{3}{|l|}{ Case II } & \multicolumn{3}{|l|}{ Case III } & \multicolumn{2}{|l|}{ Case IV } \\
\hline & $\begin{array}{l}\text { Transportation } \\
\text { Cost (\$) }\end{array}$ & $\begin{array}{l}\text { Sediment } \\
\text { (ton) }\end{array}$ & $\begin{array}{l}\text { Transportation } \\
\text { Cost (\$) }\end{array}$ & $\begin{array}{l}\text { Sediment } \\
\text { (ton) }\end{array}$ & $\begin{array}{l}\text { Constraint } \\
\text { (ton) }\end{array}$ & $\begin{array}{l}\text { Transportation } \\
\text { Cost (\$) }\end{array}$ & $\begin{array}{l}\text { Sediment } \\
\text { (ton) }\end{array}$ & $\begin{array}{l}\text { Constraint } \\
\text { (ton) }\end{array}$ & $\begin{array}{l}\text { Transportation } \\
\text { Cost (\$) }\end{array}$ & $\begin{array}{l}\text { Sediment } \\
\text { (ton) }\end{array}$ \\
\hline 1 & $2,106,900$ & 2,497 & $2,198,980$ & 2,115 & 2,163 & $2,315,270$ & 1,794 & 1,829 & $3,104,360$ & 1,495 \\
\hline 2 & 423,509 & 1,280 & 474,734 & 883 & 1,133 & 548,225 & 907 & 987 & 714,324 & 841 \\
\hline 3 & $2,448,110$ & 3,818 & $2,450,510$ & 3,357 & 3,465 & $2,595,800$ & 3,080 & 3,112 & $3,446,420$ & 2,759 \\
\hline 4 & 296,260 & 1,665 & 283,456 & 1,240 & 1,369 & 287,040 & 1,056 & 1,073 & 403,790 & 777 \\
\hline 5 & 480,781 & 3,520 & 478,513 & 2,940 & 3,186 & 523,338 & 2,805 & 2,852 & 832,801 & 2,518 \\
\hline 6 & 277,646 & 2,653 & 298,675 & 2,058 & 2,441 & 294,173 & 2,060 & 2,229 & 326,629 & 2017 \\
\hline 8 & 68,640 & 2,131 & 71,696 & 1,786 & 1,887 & 71,545 & 1,625 & 1,642 & 77,447 & 1,397 \\
\hline 9 & 45,243 & 2,863 & 45,634 & 2,114 & 2,357 & 45,482 & 1,545 & 1,852 & 54,421 & 1,347 \\
\hline 10 & 20,410 & 1,695 & 21,638 & 1,550 & 1,564 & 22,502 & 1,382 & 1,433 & 28,373 & 1,301 \\
\hline Total & $6,184,759$ & 22,791 & $6,341,404$ & 18,375 & 20,101 & $6,721,263$ & 16,636 & 17,411 & $9,007,217$ & 14,721 \\
\hline
\end{tabular}

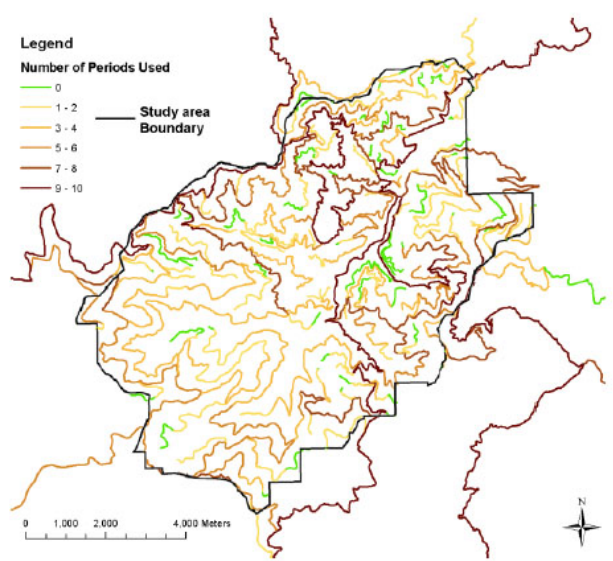

(a)

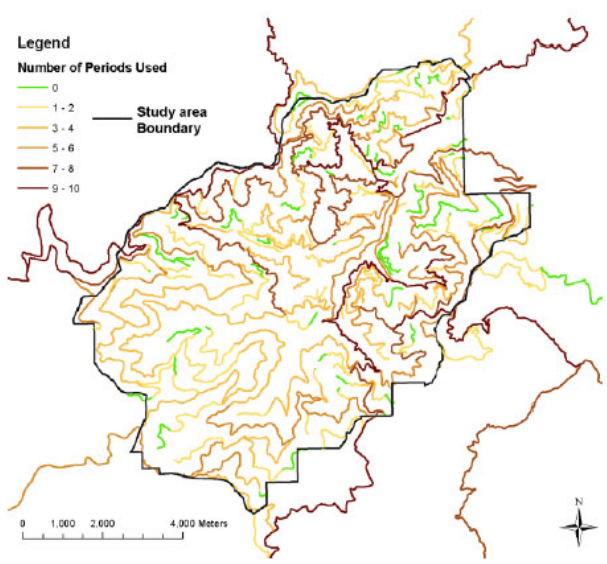

(c)

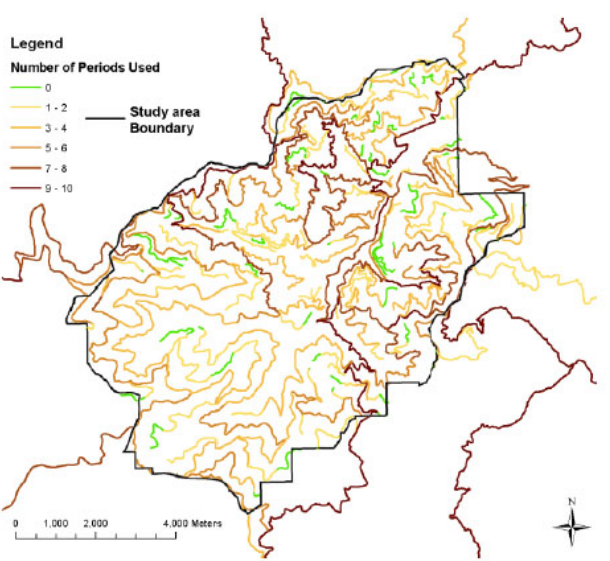

(b)

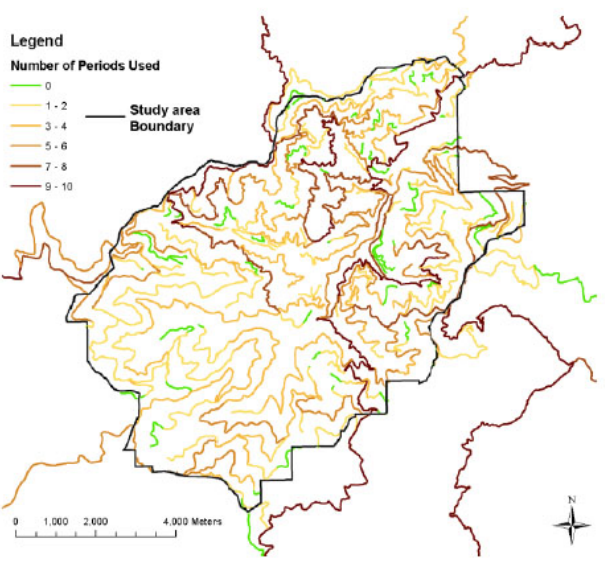

(d)

Figure 2. Best found ACO solutions showing number of periods each road segment was included in the selected routes 
For each case, the resulting solution involves a set of routes from sale locations to selected destination mills for each period. To avoid showing 40 sets of routes (one for each period and for each case), we combined routes for all ten periods into a single map showing the number of periods each road segment would be in use (see Figure 2). In general, ACOLS solutions seem relatively similar among all four cases. This is because timber sale locations are distributed across the study area (see Figure 1c), and feasible solution must include routes from all timber sales to mills regardless of restriction level. However, traffic level for each road segment, in terms of the number of periods in use, is different among all four cases. These results are also useful to identify unnecessary roads, indicating no construction in case of proposed roads or candidates for decommissioning in case for existing roads Although the ACOLS algorithm was able to find feasible solutions for all four test cases, solution quality could not be determined for this real-world, large-scale problem because of the problem size. A MIP formulation would require 199,000 variables and 259,160 constraints, thus MIP solver was not applied.

ACOLS solution quality was highly sensitive to parameter values. Thus, high-quality solutions were found only after conducting a full factorial search. Although the ACOLS algorithm was empirically proven to consistently provide high-quality results in this study, the algorithm parameters used were developed from a complete enumeration procedure conducted on the hypothetical medium-scale FTPP. It might be impractical to perform a similar search for large problems. Future work is needed to develop and apply more sophisticated techniques to efficiently identify best parameter values to ensure solution quality.

Our ACOLS provides an analytical approach that enables forest managers to directly integrate environmental impacts into the selection of cost efficient routes for timber transport. Although, we considered sediment amount, which could have a significant effect on stream water quality, our approach serve as a framework to incorporate additional environmental concerns into forest transportation planning. Additionally, the ACOLS's ability to efficiently obtain near-optimal solutions allows managers to generate alternative transportation routes and conduct sensitivity analysis to better understand the effect of sediment constraint levels on total transportation costs. Lastly, the ACOLS can provide an objective approach to determine environmental costs associated with restricting the sediment amount by comparing resulting transportation costs with the unrestricted case.

\section{CONClusions AND FUTURE WORK}

We developed a ACOLS approach to solve large-scale FTPPs considering fixed and variable costs as well as side constraints. The ability to incorporate side constraints into FTPPs facilitates addressing environmental concerns that are otherwise difficult to consider with existing tools. Computerized approaches, such as the ACOLS developed in this study, able to efficiently solve complex, large-scale problems are needed as land managers often need to address road network systems and timber transportation issues at a large landscape scale for multiple time periods under a variety of management objectives.

The ACOLS was able to match optimal MIP solutions or find near-optimal solution for all instances and cases of the hypothetical FTPP. In general, ACOLS solutions were satisfactory, but the optimality slightly decreased as sediment constraint became stricter. Trade-offs were observed between total costs of road construction and timber transportation and total amount of sediment delivery from the road network.

Setting up the appropriate parameters is important to maintain high performance of the ACOLS, and such parameters can vary not only with the size and type of problems, but also with different constraint levels. ${ }^{[7]}$ The high sensitivity of the solution quality to algorithm parameter values certainly poses a limitation of the algorithm. Future research should develop an intelligent way to identify best parameter values and also incorporate parallelism into the algorithm to further improve solution time. The need to address road management issues in spatial and temporal context at a large scale has been increasing as social and environmental demands and concerns increase around forest resources management. ${ }^{[26]}$ ACOLS has certainly the potential to provide a useful and efficient tool to meet such needs.

\section{REFERENCES}

[1] Greulich F. Transportation networks in forest harvesting: early development of the theory. Proceedings of S. 2003; 3: 57-65.

[2] Forsberg M, Frisk M, R*onnqvisty M. Flowopt-a decision support tool for strategic and tactical transportation planning in forestry. International Journal of Forest Engineering. 2005; 16 (2): 101-14.

[3] Steinberg DI. The fixed charge problem. Naval Research Logistics
Quarterly. 1970; 17 (2); 217-35.https://doi.org/10.1002/na v. 3800170209

[4] Kowalski K. On the structure of the fixed charge transportation problem. International Journal of Mathematical Education in Science and Technology. 2005; 36 (8): 879-88. https ://doi.org/10.1080/ 00207390500137837

[5] Adlakha V, Kowalski K. A simple heuristic for solving small fixed- 
charge transportation problems. Omega. 2003; 31 (3): 205-11. https ://doi.org/10.1016/S0305-0483(03)00025-2

[6] Martell DL, Gunn EA, Weintraub A. Forest management challenges for operational researchers. European Journal of Operational Research. 1998; 104 (1): 1-17. https://doi.org/10.1016/S037 7-2217 (97) 00329-9

[7] Lin P, Zhang J, Contreras MA. Automatically configuring fACOg using multilevel paramils to solve transportation planning problems with underlying weighted networks. Swarm and Evolutionary Computation. 2015; 20: 48-57. https://doi.org/10.1016/j.swev 0.2014 .10 .006

[8] Lin P, Zhang J, Contreras MA. Applying pareto ant colony optimization to solve bi-objective forest transportation planning problems. In: Information Reuse and Integration (IRI). 2014 IEEE 15th International Conference. 2014 Aug: 795-802. https://doi.org/10.1 109/iri.2014.7051970

[9] Schnelle W. A brief guide to mincost(cssg* rilib. mincst2). USDA For. Serv., Northern Region Engineering, Missoula, Mont; 1977.

[10] Weintraub A, Dreyfus S. Modifications and extensions of heuristics for solving resource transportation problems. coop agreement final report. University of California, Berkeley; 1985.

[11] Chung W, Sessions J. Network 2000, a program for optimizing large fixed and variable cost transportation problems. In: Systems Analysis in Forest Resources. Springer. 2003: 109-20. https : //doi.org/10.1007/978-94-017-0307-9_12

[12] Chung W, Sessions J. Network 2001-transportation planning under multiple objectives. In: Proceedings of the International Mountain Logging and 11th Pacific Northwest Skyline Symposium. 2001: 10-2. PMid:11263963.

[13] Grace III JM, Clinton BD. Protecting soil and water in forest road management. Transactions of the ASABE. 2007; 50 (5): 1579-84 https://doi.org/10.13031/2013.23969

[14] Rackley J, Chung W. Incorporating forest road erosion into forest resource transportation planning: a case study in the mica creek watershed in northern idaho. Transactions of the ASABE. 2008; 51 (1): 115-27. https://doi.org/10.13031/2013. 24232
[15] Efta JA. A methodology for planning road best management practices combining wepp: Road erosion modeling and simulated annealing optimization. Ph.D. thesis, The University of Montana Missoula; 2009.

[16] Dorigo M, Birattari M, St"utzle T. Ant colony optimization. Computational Intelligence Magazine, IEEE. 2006; 1(4): 28-39. https : //doi .org/10.1109/MCI . 2006. 329691

[17] Dorigo M, Blum C. Ant colony optimization theory: A survey. Theoretical Computer Science. 2005; 344(2): 243-78. https ://doi .or g/10.1016/j.tcs.2005.05.020

[18] Stutzle T, Hoos H. The max-min ant system and local search for combinatorial optimization problems. In: Meta-heuristics. Springer. 1999: 313-29.

[19] Gambardella LM, Dorigo M. An ant colony system hybridized with a new local search for the sequential ordering problem. INFORMS Journal on Computing. 2000; 12 (3): 237-55. https : //doi.org/10.1287/ijoc.12.3.237.12636

[20] L'opez-Ib'a nez M, St"utzle T. Automatic configuration of multiobjective aco algorithms. In: Swarm Intelligence. Springer. 2010: 95-106. https : //doi .org/10.1007/978-3-642-15461-4_9

[21] Contreras MA, Chung W, Jones G. Applying ant colony optimization metaheuristic to solve forest transportation planning problems with side constraints. Canadian Journal of Forest Research. 2008; 38 (11): 2896-910. https://doi.org/10.1139/X08-126

[22] Lin P. Constrained forest transportation planning problems; 2013.

[23] CPLEX II. V12. 1: Users manual for cplex. International Business Machines Corporation. 2009; 46 (53): 157.

[24] Gaertner D, Clark KL. On optimal parameters for ant colony optimization algorithms. In: IC-AI. Citeseer. 2005: 83-9.

[25] St utzle T, L'opez-Ib'anez M, Pellegrini P, et al. Parameter adaptation in ant colony optimization. In: Autonomous search. Springer. 2011: 191-215. https ://doi.org/10.1007/978-3-642-21434-9_8

[26] Lin P, Contreras MA, Dai R, et al. A multilevel fACOg approach for solving forest transportation planning problems with environmental constraints. Swarm and Evolutionary Computation. 2016; 28: 78-87. https://doi.org/10.1016/j.swevo.2016.01.003 